Check for updates

Cite this: RSC Adv., 2017, 7, 19169

Received 16th January 2017

Accepted 19th March 2017

DOI: $10.1039 / \mathrm{c} 7 \mathrm{ra00672a}$

rsc.li/rsc-advances

\section{Polarization independent and tunable plasmon induced transparency for slow light}

\author{
Hanning Yang, (D) ab Edgar Owiti, ${ }^{\text {ab }}$ Yanbo Pei, ${ }^{\text {ab }}$ Siren Li, ${ }^{\text {ab }}$ Peng Liu ${ }^{\text {ab }}$ \\ and Xiudong Sun (ID *ab
}

A novel metamaterial composed of a Ag nanoprism periodic tetramer is proposed in this paper. The metamaterial has high structural symmetry and shows polarization independent plasmon-induced transparency (PIT), demonstrated through simulation based on a finite element method. Resonant wavelength and transmissivity of the transparency window is flexibly tuned by changing the fillet radius, the edge length, and the thickness of the Ag nanoprisms. A slow-light effect, caused by an adjustable PIT with a special line shape, can also be flexibly manipulated as the nanoprisms are filleted. The maximum group index value of the metamaterial ranges from 71 to 225. The effects show potential for application in novel plasmonic environmental sensors and slow-light devices.

\section{Introduction}

Metal metamaterials have attracted extensive attention due to their unique optical properties such as a negative refractive index, ${ }^{\mathbf{1 , 2}}$ slow-light, ${ }^{\mathbf{3}, 4}$ and Fano resonance. ${ }^{5}$ Plasmoninduced transparency (PIT), as an exceptional case of Fano resonance in a metamaterial, is an analogy of atomic electromagnetically induced transparency (EIT) effect. ${ }^{6-8}$ The PIT effect can be attributed to the destructive interference between two resonances with different line widths. Compared with the typical atomic EIT effect, the PIT effect in a metal metamaterial possesses a low requirement to the operation environment ${ }^{8}$ and for state-of-the-art preparation. ${ }^{9}$ The application of the PIT device has more practically essential significance. Due to its planar structure and the sharp line shape of the PIT effect, metal metamaterials have various practical applications that include plasmonic sensing ${ }^{10}$ and optical switching. ${ }^{\mathbf{1 1 - 1 3}}$

The PIT effect in metal metamaterials together with its sharp line shape can cause a slow-light effect. ${ }^{\mathbf{1 4 - 1 6}}$ Because of the relatively large intrinsic loss of metal in metamaterial, the maximum value of its group index is usually less than 100 . The performance of the slow-light effect caused by PIT effect can also be flexibly modulated through changing the shapes and structural parameters of the unit cell in metamaterial. ${ }^{17-19}$ Metamaterials composed of identical and symmetrically arranged resonators have been investigated and shown polarization independent optical properties. ${ }^{\mathbf{2 0 - 2 3}}$

${ }^{a}$ Department of Physics, Harbin Institute of Technology, Harbin, 150001, P. R. China. E-mail:xdsun@hit.edu.cn

${ }^{b}$ Key Lab of Micro-Optics and Photonic Technology of Heilongjiang Province, Harbin, 150001, P. R. China
Metal nanoprisms with tunable dipolar and quadrupolar surface plasmons have attracted wide attentions in the past decade. $^{\mathbf{2 4 , 2 5}}$ Recently, metal nanoprisms with unique structural features (high structural symmetry and strong anisotropy) and easy preparation characteristic have become important building blocks for metal planar metamaterial. ${ }^{26,27}$ However, optical properties of the planar metamaterials composed of multiple metal nanoprisms still need to be further investigated. Such optical properties are usually nonadjustable once they are prepared, which is a huge limitation to their utility. Various works that involve replacing the conventional materials in metamaterials with the phase change materials and the materials with adjustable permittivity, ${ }^{28-33}$ have all been demonstrated to break the presented limitation. Since Ag metal is quite sensitive to its surrounding environment, an Ag nanoprism can be easily filleted and its optical response manipulated accordingly. ${ }^{34-36}$ This provides a new modulation mechanism on the optical properties of the metamaterials.

In this paper, the optical property of the planar metamaterial consisting of $\mathrm{Ag}$ nanoprism periodic tetramer is theoretically studied. The metamaterial exhibits a PIT effect that is independent of the polarization direction of incident light and slightly dependent upon the angle of rotation of Ag nanoprism. The PIT effect is flexibly tuned through varying the fillet radius, the edge length, and the thickness of the Ag nanoprism. The planar metamaterial also shows a flexible slow-light effect and a maximum value of group index that could reach 225 upon varying the fillet radius of $\mathrm{Ag}$ nanoprism. These results indicate the potential of Ag planar metamaterial with tunable PIT and slow-light effects as a candidate for novel plasmonic environmental sensor and slow-light nano-devices. 


\section{Structure model and methods}

The unit cell of the planar metamaterial proposed this paper is schematized in Fig. 1. In the unit cell, four identical nanoprisms (grey) with same structural parameters were placed on silica substrate (purple). The surface of the silica substrate is parallel to the $x-y$ plane, the nanoprism tetramers are periodic in the $x$ and $y$ directions, and the whole structure is covered by air $(n=1)$. A linearly polarized light propagates along the negative direction of $z$-axis and vertically illuminates the surface of the metamaterial.

The optical properties of the metamaterials under different polarized incident light were considered. As shown in Fig. 1(a), the angle between the polarization direction of the incident light and $x$-axis, defined as the polarization angle $\theta$ was varied from $0^{\circ}$ to $90^{\circ}$. The rotation angle $\beta$ is the angle between the base of $\mathrm{Ag}$ nanoprism and $x$-axis. $\mathrm{Ag}$ nanoprism tetramer with $\beta$ equal to $90^{\circ}$ is the same as that for $\beta$ equal to $0^{\circ}$, therefore the period of rotation of $\mathrm{Ag}$ nanoprism tetramer in unit cell was taken as $90^{\circ}$. To study the impact of the rotation angle, the optical properties of metamaterials with $\beta$ ranging from $0^{\circ}$ to $90^{\circ}$ are herein investigated.

The influence of the surrounding environment to the planar metamaterial was characterized by changing the fillet radius of $\mathrm{Ag}$ nanoprism $(r)$. The other geometric parameters in unit cell are the thickness $(h)$ and the edge length $(L)$ of Ag nanoprism. The Ag nanoprism periodic tetramer possesses a square lattice, and the periods along $x$-axis and $y$-axis $(P)$ are fixed at $300 \mathrm{~nm}$. In particular, the rotation angle, the fillet radius, the edge length, and the thickness were manipulated to investigate their influences on the optical properties of the metamaterials.

Numerical simulations were performed using Comsol Multiphysics. The material of nanoprism in the planar metamaterial was chosen as Ag, its frequency dependent refractive index function was obtained from the experimental data of Johnson and Christy. ${ }^{37}$ The material of the substrate was silica, and its dielectric function was from Palik. ${ }^{38}$ The wavelength of the incident light was varied from $500 \mathrm{~nm}$ to $1000 \mathrm{~nm}$ with intermediate steps of $4 \mathrm{~nm}$, moreover, the step was fine tuned to $1 \mathrm{~nm}$ at resonant wavelength.

The group index of the planar metamaterial $\left(n_{\mathrm{g}}\right)$ was calculated in the following form..$^{7,39,40}$

$$
n_{\mathrm{g}}=n_{\text {eff }}-\lambda \frac{\mathrm{d} n_{\text {eff }}}{\mathrm{d} \lambda}
$$
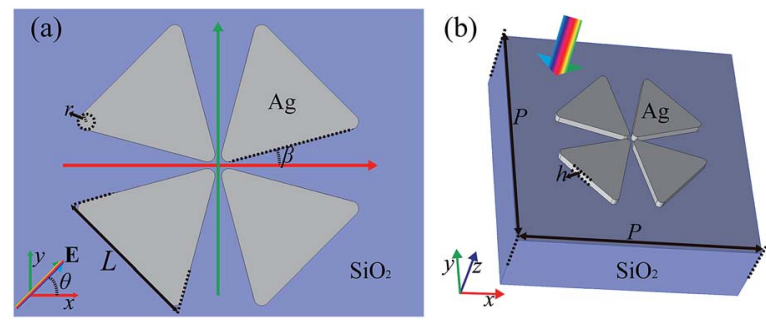

Fig. 1 Vertical-view (a) and tilt-view (b) schematic illustration of Ag nanoprism tetramer with specified structural parameters $\left(\beta=15^{\circ} ; \theta=\right.$ $45^{\circ} ; L=100 \mathrm{~nm}, h=10 \mathrm{~nm}, r=10 \mathrm{~nm}, P=300 \mathrm{~nm}$ ). where $n_{\text {eff }}$ is the effective refractive index of the metamaterial, $\lambda$ is the wavelength of the incident light. The effective refractive index was calculated from the scattering $(S)$ matrix in the following form. ${ }^{40}$

$$
n_{\mathrm{eff}}=\frac{1}{k h} \cos ^{-1}\left[\frac{1}{2 S_{21}}\left(1-S_{11}^{2}+S_{21}^{2}\right)\right]
$$

where $h$ is the thickness of Ag nanoprism, $S_{11}$ and $S_{21}$ are the reflection and transmission coefficients, $k$ is the wave vector of the incident light.

\section{Simulation results and discussion}

The optical properties of the metamaterials versus the polarization direction of the incident light were firstly investigated as shown in Fig. 2. The metamaterial with $\beta$ equal to $0^{\circ}$ shows a typical PIT effect in Fig. 2(a). The resonant wavelengths of the transparency window (peak1, $765 \mathrm{~nm}$ ) and the two dips (dip1, $708 \mathrm{~nm}$; and dip2, $814 \mathrm{~nm}$ ) in Fig. 2(a) are kept unchanged as $\theta$ ranges from $0^{\circ}$ to $90^{\circ}$. The transmissivity of peak1 is a constant $(0.873)$ and also independent on the polarization.

The PIT effect can be analysed by the surface charge distributions of the metamaterial at the resonant wavelengths. As shown in Fig. 2(b), the charge distribution of dip1 (A) displays an apparent dipole resonance distribution with $\theta=45^{\circ}$. The charge distribution of dip2 (C) shows a typical quadrupole resonance distribution. The distribution of peak1 (B) possesses the distribution characteristics of the two dips, which means that the transparency window is a result of destructive interference of dip1 (the dipole resonance) and dip2 (the quadrupole resonance). Due to the rotational symmetry of $\mathrm{Ag}$ nanoprism tetramer in metamaterial, the charge distribution varies from one nanoprism to the adjacent nanoprism as $\theta$ is increased. The effective resonant lengths of the dipole and quadrupole resonances remain almost unchanged versus the polarization, so the resonant wavelengths and transmissivity are nearly constants. The planar metamaterial shows a PIT effect, which is independent of the polarization direction of the incident light.

Fig. 3 shows the resonant wavelengths and transmissivity distributions of the PIT effect versus $\theta$ and the rotation angle of $\mathrm{Ag}$ nanoprism $(\beta)$. The step of the incident light wavelength here

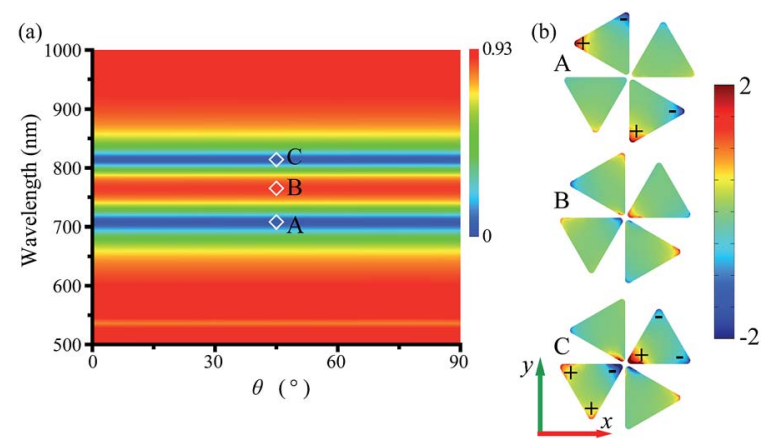

Fig. 2 (a) Transmission map versus $\theta$ and wavelength. (b) Surface charge distributions at different points (A: dip1; B: peak1; C: $\operatorname{dip} 2 ; \theta$ : $\left.45^{\circ}\right)$. 

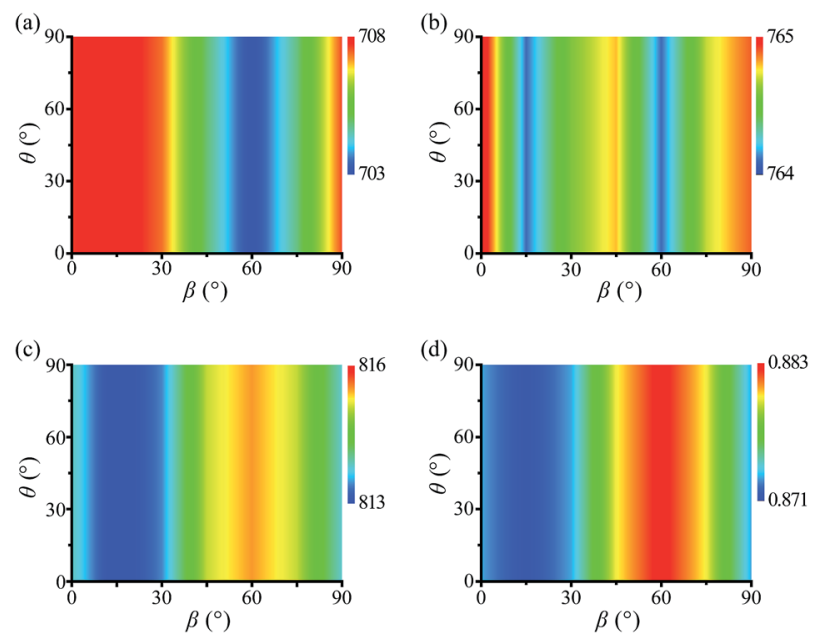

Fig. 3 Resonant wavelength maps versus $\theta$ and $\beta$; (a), (b) and (c) are dip1, peak1 and dip2, respectively. (d) Resonant intensity maps of peak1.

is $0.1 \mathrm{~nm}$. As shown in Fig. 3(a) and (c), the resonant wavelengths of the two dips show opposite variation tendencies against $\beta$. The dip1 has a maximum value $(708.2 \mathrm{~nm})$ with $\beta$ equal to $15^{\circ}$, and dip2 has a minimum value $(812.8 \mathrm{~nm})$. However, dip1 has a minimum value $(702.9 \mathrm{~nm})$ with $\beta$ equal to $60^{\circ}$, and dip2 has a maximum $(815.5 \mathrm{~nm})$. The variation range of dip1 is $5.3 \mathrm{~nm}$, and that of dip2 is $2.7 \mathrm{~nm}$. This can be attributed to the fact that the structural symmetry of the tetramer changes are caused by the variation of $\beta$. The nanoprism tetramers possess mirror symmetry relative to $x-z$ plane and $y-z$ plane with $\beta$ equal to $15^{\circ}$ and $60^{\circ}$. This leads to the small changes of the effective lengths of the dipole and quadrupole resonances. The small variations in the effective lengths lead to a little shift of the two dips.

The resonant wavelengths of the transparency window in Fig. 3(b) have two minimum values $(764.1 \mathrm{~nm})$ when $\beta$ is equal to $15^{\circ}$ and $60^{\circ}$, respectively. It is worth noting that the wavelength variation range of peak1 is less than $1 \mathrm{~nm}$ which is less than the difference between the variations of the two dips. This indirectly confirms that peak 1 is caused by destructive interference of the two dips. The transmissivity of peak1 in Fig. 3(d) has a sine-like variation against $\beta$. The minimum point of the transmissivity is at $15^{\circ}$, and the maximum at $60^{\circ}$, with 0.01 variation range. The planar metamaterial with an arbitrary rotation angle in Fig. 3(a-c) shows an invariable PIT effect versus the polarization. The PIT effect of the metamaterial is independent of the polarization of the incident light, and slightly dependent upon the rotation angle of Ag nanoprism.

As mentioned before, the tips of Ag nanoprisms are flexibly filleted due to the oxidation of Ag atom caused by a change of its surrounding. $\mathrm{Ag}$ nanoprism gradually transforms into $\mathrm{Ag}$ nanodisk as it is filleted, its optical property accordingly changes. So the studies on the influences of the radius of $\mathrm{Ag}$ nanoprism on the PIT effect become more significant. The results are shown in Fig. 4. As $r$ is increased, the transparency window in Fig. 4(a) weakens and narrows down. The peak1 in
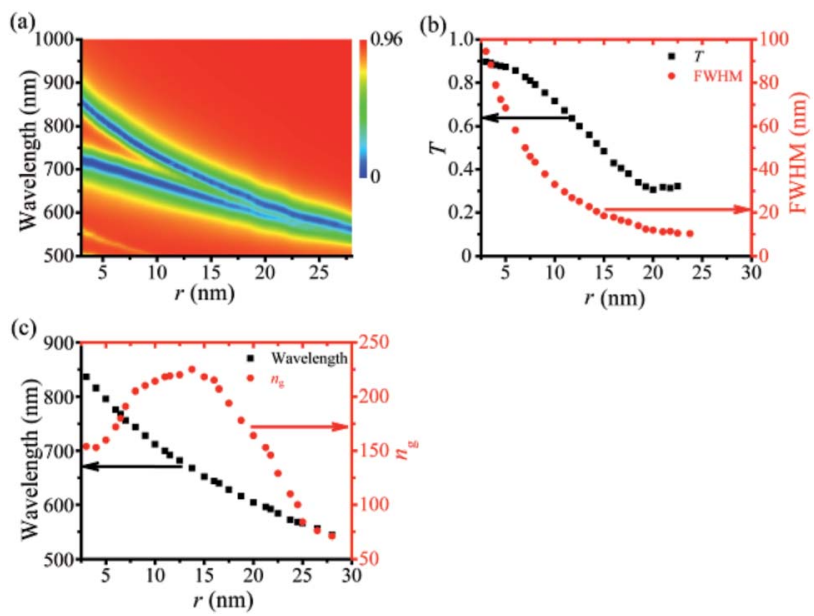

Fig. 4 (a) Transmission map versus $r$ and wavelength. (b) FWHM and transmissivity ( $T$ ) spectra of peak1. (c) Resonant wavelength and intensity $\left(n_{\mathrm{g}}\right)$ spectra of the group index versus $r$.

Fig. 4(a) shows a linear blue-shift effect against $r$. The transmissivity in Fig. 4(b) exponentially decays from 0.9 to 0.3 , and then peak1 disappears. The distance between nanoprisms in unit cell increases as the tips of $\mathrm{Ag}$ nanoprisms are filleted, the interaction strength of the destructive interference weakens accordingly. When $r$ reaches a critical value, the distance between nanoprisms in the unit cell are far enough and the interaction between the two resonances disappears accordingly, and peak1 vanishes.

The full width half maximum (FWHM) of peak1 in Fig. 4(b) exponentially decreases as $r$ is increased. This also can be attributed to the weakening interaction between the two resonances as the $\mathrm{Ag}$ nanoprisms in unit cell are filleted. So peak1 which is caused by the destructive interference of the two resonances fades. Therefore, the resonant wavelengths and transmissivity of the PIT effect can be modulated by changing the fillet radius of $\mathrm{Ag}$ nanoprism. Due to the linear shift of peak1 versus the fillet radius of $\mathrm{Ag}$ nanoprism (i.e. its surrounding environment), the planar metamaterial can be a potential environmental sensitive sensor.

The planar metamaterial also shows an obviously slow-light effect caused by the sharp line shape of its PIT effect. Due to the tunability of the PIT effect, its group index can also be flexibly tuned by manipulating the fillet radius of Ag nanoprism as shown in Fig. 4(c). The resonant wavelength of the group index shows a blue-shift effect. The resonant wavelength varies from $836 \mathrm{~nm}$ to $544 \mathrm{~nm}$. Its intensity firstly strengthens and then weakens as $r$ increases. The group index is greater than 200 with $r$ ranging from 7.5 to $17.5 \mathrm{~nm}$. The group index reaches 225 when $r=13.75 \mathrm{~nm}$, and its minimum value is 71 when $r=3 \mathrm{~nm}$. This result means that the planar metamaterial composed of Ag filleted nanoprism tetramer also can be a candidate for the flexibly tunable slow-light device as Ag nanoprism is filleted (i.e., a change of the surrounding). This effect overcome a common obstacle that group indices of the metamaterials are fixed once the metamaterials are prepared. 

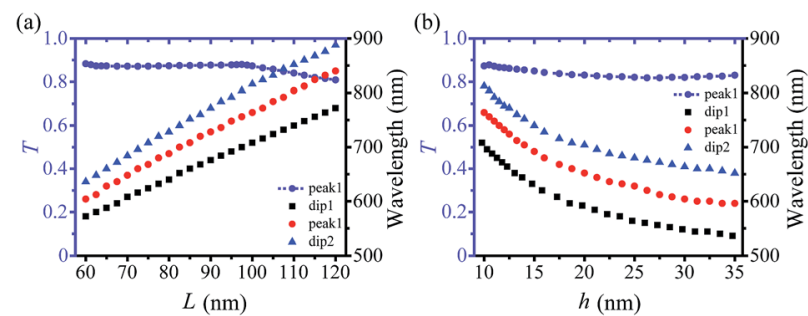

Fig. 5 Resonant wavelength and transmissivity spectra versus the edge length (a) and the thickness (b) of Ag nanoprism.

The impacts of the edge length and thickness of Ag nanoprism on the PIT effect of the planar metamaterial are shown in Fig. 5. The transmissivity of peak1 in Fig. 5(a) is kept almost unchanged (0.9) when $L<95 \mathrm{~nm}$, while when $L>95 \mathrm{~nm}$ the transmissivity linearly decreases gradually. This can be attributed to the fact that the interval between nanoprisms is kept unchanged as $L$ increases. The interaction strength between the two resonances remains the same, the transmissivity therefore also stay the same. The two dips and peak1 show a linear redshift effect against $L$. The variation range of the resonant wavelength of peak1 is as large as $300 \mathrm{~nm}$, while the variation of $L$ is $60 \mathrm{~nm}$. This can be attributed to that the effective resonant lengths of the dipole and quadrupole resonances increase as $L$ increases. The PIT effect of the planar metamaterial can be flexibly modulated in visible region without loss of its transmissivity by changing the edge length of $\mathrm{Ag}$ nanoprism.

The resonant wavelengths of the PIT effect in Fig. 5(b) exponentially decay as the thickness of $\mathrm{Ag}$ nanoprism is increased from $10 \mathrm{~nm}$ to $35 \mathrm{~nm}$ and slightly decrease when $h>$ $30 \mathrm{~nm}$. This is similar to the variation of the resonance of single $\mathrm{Ag}$ nanoprism relative to the change of $h$. The transmissivity gradually becomes weaker, with a small variation relative to the value of transmissivity. The resonant wavelengths of the PIT effect can be flexibly tuned, and its transmissivity slightly changed through modulating the thickness of Ag nanoprism. The PIT effect of the metamaterial with specific wavelength in visible-infrared region can be realized by choosing a specific edge length and thickness. So the slow light originating from the PIT effect can also be tuned by the edge length and thickness. The group index increases as $L$ increases, and decreases as $\mathrm{Ag}$ nanoprism becomes thicker. This metamaterial can be a potential plasmonic device in visible region.

\section{Conclusions}

In summary, $\mathrm{Ag}$ metamaterial composed of periodic nanoprism tetramer proposed in this paper shows an apparent PIT effect. The PIT effect originates from the destructive interference of the dipole and quadrupole resonances. Due to the special structural symmetry, the PIT effect is independent of the polarization angle of the incident light, and slightly dependent on the rotation angle of $\mathrm{Ag}$ nanoprism. The PIT and slow-light effects can be flexibly modulated through changing the fillet radius of $\mathrm{Ag}$ nanoprism. The resonant wavelengths of the PIT effect can also be flexibly tuned in the visible-infrared region through manipulating the edge length and thickness of $\mathrm{Ag}$ nanoprism in metamaterial. This research paves way for designing novel plasmonic environmental sensors, plasmonic devices, and tunable slow-light devices.

\section{Acknowledgements}

This work was funded by the National Natural Science Foundation of China (No. 11374074) and the National Basic Research Program of China (No. 2013CB328702).

\section{Notes and references}

1 R. A. Shelby, D. R. Smith and S. Schultz, Science, 2001, 292, 77-79.

2 S. A. Ramakrishna, Rep. Prog. Phys., 2005, 68, 449-521.

3 H. Gersen, T. J. Karle, R. J. Engelen, W. Bogaerts, J. P. Korterik, N. F. van Hulst, T. F. Krauss and L. Kuipers, Phys. Rev. Lett., 2005, 94, 073903.

4 J. Gu, R. Singh, X. Liu, X. Zhang, Y. Ma, S. Zhang, S. A. Maier, Z. Tian, A. K. Azad, H. T. Chen, A. J. Taylor, J. Han and W. Zhang, Nat. Commun., 2012, 3, 1151.

5 B. Luk'yanchuk, N. I. Zheludev, S. A. Maier, N. J. Halas, P. Nordlander, H. Giessen and C. T. Chong, Nat. Mater., 2010, 9, 707-715.

6 C. Kurter, P. Tassin, L. Zhang, T. Koschny, A. P. Zhuravel, A. V. Ustinov, S. M. Anlage and C. M. Soukoulis, Phys. Rev. Lett., 2011, 107, 043901.

7 S. Biswas, J. Duan, D. Nepal, K. Park, R. Pachter and R. A. Vaia, Nano Lett., 2013, 13, 6287-6291.

8 J. Song, J. Liu, K. Li, Y. Song, X. Wei and G. Song, IEEE Photonics Technol. Lett., 2014, 26, 1104-1107.

9 S. Zhang, D. A. Genov, Y. Wang, M. Liu and X. Zhang, Phys. Rev. Lett., 2008, 101, 047401.

10 N. Liu, T. Weiss, M. Mesch, L. Langguth, U. Eigenthaler, M. Hirscher, C. Sonnichsen and H. Giessen, Nano Lett., 2010, 10, 1103-1107.

11 J. Chen, P. Wang, C. Chen, Y. Lu, H. Ming and Q. Zhan, Opt. Express, 2011, 19, 5970-5978.

12 Z. He, H. Li, S. Zhan, B. Li, Z. Chen and H. Xu, Sci. Rep., 2015, $5,15837$.

13 F. Zhang, X. Hu, H. Yang and Q. Gong, Appl. Phys. Lett., 2014, 104, 131110.

14 G. Wang, H. Lu and X. Liu, Appl. Phys. Lett., 2012, 101, 013111.

15 N. Liu, L. Langguth, T. Weiss, J. Kastel, M. Fleischhauer, T. Pfau and H. Giessen, Nat. Mater., 2009, 8, 758-762.

16 M. Fleischhauer, A. Imamoglu and J. P. Marangos, Rev. Mod. Phys., 2005, 77, 633-673.

17 N. Liu, M. Hentschel, T. Weiss, A. P. Alivisatos and H. Giessen, Science, 2011, 332, 1407-1410.

18 T. J. Davis, D. E. Gomez and K. C. Vernon, Nano Lett., 2010, 10, 2618-2625.

19 T. Zentgra, S. Zhang, R. F. Oulton and X. Zhang, Phys. Rev. B: Condens. Matter Mater. Phys., 2009, 80, 195415.

20 R. Singh, C. Rockstuhl, F. Lederer and W. Zhang, Phys. Rev. B: Condens. Matter Mater. Phys., 2009, 79, 085111. 
21 X. Duan, S. Chen, H. Yang, H. Cheng, J. Li, W. Liu, C. Gu and J. Tian, Appl. Phys. Lett., 2012, 101, 143105.

22 X. Duan, S. Chen, H. Cheng, Z. Li and J. Tian, Opt. Lett., 2013, 38, 483-485.

23 S. Xiao, T. Wang, Y. Liu, X. Han and X. Yan, Plasmonics, 2017, 12, 185-191.

24 J. E. Millstone, S. Park, K. L. Shuford, L. Qin, G. C. Schatz and C. A. Mirkin, J. Am. Chem. Soc., 2005, 127, 5312-5313.

25 L. J. Sherry, R. Jin, C. A. Mirkin, G. C. Schatz and R. P. V. Duyne, Nano Lett., 2006, 6, 2060-2065.

26 C. Lu, X. Hu, K. Shi, Q. Hu, R. Zhu, H. Yang and Q. Gong, Light: Sci. Appl., 2015, 4, e302.

27 C. Lu, X. Hu, F. Zhang, H. Yang and Q. Gong, Adv. Opt. Mater., 2014, 2, 1141-1148.

28 P. Pitchappa, M. Manjappa, C. P. Ho, R. Singh, N. Singh and C. Lee, Adv. Opt. Mater., 2016, 4, 541-547.

29 X. Zhao, L. Zhu, C. Yuan and J. Yao, Opt. Lett., 2016, 41, 5470. 30 Z. Fang, Y. Wang, A. E. Schlather, Z. Liu, P. M. Ajayan, F. J. de Abajo, P. Nordlander, X. Zhu and N. J. Halas, Nano Lett., 2014, 14, 299-304.
31 H. Cheng, S. Chen, P. Yu, W. Liu, Z. Li, J. Li, B. Xie and J. Tian, Adv. Opt. Mater., 2015, 3, 1744-1749.

32 H. Cheng, S. Chen, P. Yu, X. Duan, B. Xie and J. Tian, Appl. Phys. Lett., 2013, 103, 203112.

33 H. Cheng, S. Chen, P. Yu, J. Li, L. Deng and J. Tian, Opt. Lett., 2013, 38, 1567-1569.

34 L. Chen, X. Fu, W. Lu and L. Chen, ACS Appl. Mater. Interfaces, 2013, 5, 284-290.

35 W. Y. Ma, J. Yao, H. Yang, J. Y. Liu, F. Li, J. P. Hilton and Q. Lin, Opt. Express, 2009, 17, 14967-14976.

36 X. Yang, Y. Yu and Z. Gao, ACS Nano, 2014, 8, 4902-4907.

37 P. B. Johnson and R. W. Christy, Phys. Rev. B: Condens. Matter Mater. Phys., 1972, 6, 4370-4379.

38 E. D. Palik, Handbook of optical constants of Solids, Academic Press, 1985.

39 B. E. A. Saleh and M. C. Teich, Wiley Series in Pure and Applied Optics, USA, 1991.

40 D. R. Smith, D. C. Vier, T. Koschny and C. M. Soukoulis, Phys. Rev. E: Stat., Nonlinear, Soft Matter Phys., 2005, 71, 036617. 